\title{
Bone Degeneration and Recovery after Early and Late Bisphosphonate Treatment of Ovariectomized Wistar Rats Assessed by In Vivo Micro-Computed Tomography
}

\author{
J. E. M. Brouwers · F. M. Lambers • \\ J. A. Gasser · B. van Rietbergen · R. Huiskes
}

Received: 3 July 2007/ Accepted: 12 October 2007/Published online: 20 February 2008

(C) The Author(s) 2008

\begin{abstract}
Bisphosphonates are antiresorptive drugs commonly used to treat osteoporosis. It is not clear, however, what the influence of the time point of treatment is. Recently developed in vivo micro-computed tomographic (CT) scanners offer the possibility to study such effects on bone microstructure in rats. The aim of this study was to determine the influence of early and late zoledronic acid treatment on bone in ovariectomized rats, using in vivo micro-CT. Twenty-nine female Wistar rats were divided into the following groups: ovariectomy (OVX, $n=5$ ), OVX and zoledronic acid (ZOL) at week $0(n=8)$, OVX and ZOL at week $8(n=7)$, and sham $(n=9)$. CT scans were made of the proximal tibia at weeks $0,2,4,8,12$, and 16; and bone structural parameters were determined in the metaphysis. Two fluorescent labels were administered to calculate dynamic histomorphometric parameters. At week 16, all groups were significantly different from each other in bone volume fraction (BV/TV), connectivity density, and trabecular number (Tb.N), except for the early ZOL and control groups which were not significantly different for any structural parameter. After ZOL treatment at week 8 , BV/TV, structure model index, Tb.N, and trabecular
\end{abstract}

J. E. M. Brouwers · F. M. Lambers · B. van Rietbergen ( $\square)$. R. Huiskes

Eindhoven University of Technology, P.O. Box 513,

5600MB Eindhoven, The Netherlands

e-mail: b.v.rietbergen@tue.nl

J. E. M. Brouwers

e-mail: j.e.m.brouwers@tue.nl

J. A. Gasser

Novartis Institutes for Biomedical Research, Musculoskeletal

Diseases, WKL-125.10.15 Basel, Switzerland thickness significantly improved in the late ZOL group. The OVX and ZOL groups showed, respectively, higher and lower bone formation rates than the control group. Early ZOL treatment inhibited all bone microstructural changes seen after OVX. Late ZOL treatment significantly improved bone microstructure, although the structure did not recover to original levels. Early ZOL treatment resulted in a significantly better microstructure than late treatment. However, late treatment was still significantly better than no treatment.

Keywords Osteoporosis · Bisphosphonate - In vivo micro-computed tomography $\cdot$ Rat

Postmenopausal osteoporosis affects millions of women worldwide and results in loss of bone mass and bone microstructural changes, which lead to reduced bone strength. It remains unknown how exactly the microstructure is affected over the time course of osteoporosis development and to what extent this structure can be recovered by drug treatment. Since the assessment of bone structure in humans is still limited to a few peripheral sites, the ovariectomized rat is used in many studies as an animal model for osteoporosis in cross-sectional evaluations. Numerous cross-sectional studies have determined the loss of trabecular bone in the proximal tibia in rats following ovariectomy $(\mathrm{OVX})$ at different time points by measuring bone structural parameters [1-3]. OVX has been shown to decrease bone volume fraction, connectivity, and trabecular number and to increase trabecular separation and structure model index, which indicates that trabecular bone changes from plate-like to more rod-like in the proximal tibial metaphysis of the female rat. However, conflicting results were found for the effects of OVX on trabecular thickness, showing increases, decreases, or no response at 
all [4-6]. These cross-sectional studies are limited to comparing averages per group only, which provides a reduced sensitivity for detection of differences. In addition, no information is obtained regarding the specific locations of bone changes.

Recently, however, in vivo micro-computed tomographic (CT) scanners became available, which can monitor the process of bone loss and microstructural changes in small, living animals. So far, two studies have been reported in which this equipment was used to monitor changes in rat bones after OVX. Interestingly, these studies reported, respectively, significant increases [5] and decreases [7] in trabecular thickness after OVX, indicating that the response may vary. While the process of bone degeneration after OVX is not fully understood, even less information is available for the process of recovery after drug treatment. The most widely used drugs for treatment of diseases associated with increased bone resorption, such as postmenopausal osteoporosis, are now bisphoshonates.

Many cross-sectional studies have determined the effects of bisphosphonates in rats either as a preventive treatment, before development of OVX-induced bone loss, or as a recovering treatment after development of bone loss. Although the results are difficult to compare directly due to differences in experimental design, preventive treatment in animals may lead to the same amount of final bone mass as recovering treatment. The associated microstructure in terms of number and thickness of trabeculae, however, can be different. While the influence of the time point of treatment is highly relevant clinically, no studies were found that directly compared the effects of a preventive and a recovering treatment on final bone mass and associated microstructure. In addition, the fact that only results of cross-sectional studies have been reported complicates comparison of the final and original bone structure and provides little or no information on the changes in structure over time. Here also we expect that longitudinal in vivo micro-CT imaging will potentially provide more information about the specific changes in the bone, both spatially and temporally.

For the present study, we had two goals: (1) to determine the process of bone loss and bone microstructural changes in ovariectomized rats and (2) to determine the effects of early and late treatment of osteoporotic rats with a bisphosphonate. We used an in vivo high-resolution micro-CT scanner to image the proximal tibia of female adult Wistar rats. For the treatment, zoledronic acid (ZOL) was used, since this is a third-generation bisphosphonate that proved a potent osteoclast inhibitor. It has shown to be effective in cancer patients with bone metastases and is currently being tested in clinical trials as a promising therapeutic agent for osteoporotic patients $[8,9]$.

\section{Methods}

Animals

Thirty-six female, 30-week-old, retired breeding Wistar rats were obtained from Harlan Laboratories (Horst, The Netherlands) and allowed to acclimate for 7 days before the start of the experiment. The rats were maintained with a cycle of 12 hours light and 12 hours darkness and allowed to eat and drink ad libitum. The experiment was approved by the Animals Ethics Committee of the University of Maastricht, The Netherlands. The rats were divided into four groups based on weight: control $(n=9)$, OVX $(n=9)$, OVX and early ZOL $(n=9)$, OVX and late ZOL $(n=9)$. All rats were ovariectomized at week 0 , and the control group underwent sham OVX. Success of OVX was confirmed at necropsy by determining atrophy of the uterine horns. ZOL (kindly donated by Novartis Pharmaceutical, Basel, Switzerland) was dissolved in a saline vehicle. The early ZOL group was administered $\mathrm{ZOL}$ at a single dose of $20 \mu \mathrm{g} / \mathrm{kg}$ body weight s.c. at OVX (comparable to the phase III clinical trial dose used in osteoporotic patients). The late ZOL group was administered the same single dose 8 weeks after OVX. Eleven and 4 days before necropsy, the fluorescent labels calcein (Fluka, Buchs, Switzerland) and alizarin (Merck, Dietikon, Switzerland) were administered s.c. in a saline vehicle to all rats at doses of $30 \mathrm{mg} / \mathrm{kg}$ and $20 \mathrm{mg} / \mathrm{kg}$ body weight, respectively, to evaluate bone formation dynamics in the proximal tibial metaphysis. Rats were killed at 16 weeks by exsanguination.

\section{Micro-CT Scanning}

Directly after the operation, a 6-mm micro-CT scan (70 kV, $85 \mu \mathrm{A}, 1,000$ projections per 180 degrees, $350 \mathrm{~ms}$ integration time) with an isotropic resolution of $15 \mu \mathrm{m}$ was made of the proximal tibia using an in vivo micro-CT scanner (vivaCT 40; Scanco Medical, Bruettisellen, Switzerland). The CT scanner was calibrated, and a beamhardening correction algorithm was applied to all scans. Follow-up in vivo CT scans were made after 2, 4, 8, 12, and 16 weeks to monitor bone structure. One CT scan took 35 minutes, during which the animal was anesthetized with isoflurane and the scanned leg was placed in a custommade leg-fixating device. The design of the rat holder was such that the left leg was not exposed to radiation while scanning the right leg. Radiation damage to the scanned bone was not expected to occur, based on a previous study in which 8-weekly CT scans with the same radiation dose caused no detected bone damage [10]. In that study, we also showed that the reproducibility of all structural 
parameters was high, with a coefficient of variation of about $1 \%$.

Image processing included gaussian filtering and segmentation and was described elsewhere in detail [10]. In brief, the same filtering and segmentation values were used for every measurement of each animal (sigma $=0.7$, support $=1$, threshold density $=0.504 \mathrm{~g}$ hydroxyapatite/cc, equivalent to $22 \%$ of maximal gray-scale value). From every baseline and follow-up CT scan, the metaphyseal trabecular bone was manually selected and bone structural parameters (bone volume fraction [BV/TV], connectivity density [Conn.D], structure model index [SMI], trabecular number [Tb.N], trabecular thickness [Tb.Th], and trabecular separation $[\mathrm{Tb} . \mathrm{Sp}]$ ) were automatically determined. The average attenuation coefficient of the trabecular bone tissue was determined for all measurements using a protocol provided by the manufacturer of the micro-CT scanner. With this protocol, the gray levels of voxels near the trabecular surfaces are not included, to ensure that the measurements are not affected by partial volume effects. Also, cortical thickness of the metaphysis was analyzed. The cortical bone starting from the tibia-fibula junction was manually selected for each measurement, resulting in a region approximately $1.8 \mathrm{~mm}$ long. The same filtering and segmentation values were used as for the trabecular analysis. Cortical thickness was automatically determined from the selected region.

When two CT scans of the same animal are made at different time points, the position of the animal in the scanner will not be exactly the same and, therefore, the CT scans will also differ. In order to detect bone structural changes on a micro level, one CT scan needs to be translated and rotated to match the other. We developed image registration software that registers two scans based on minimizing the correlation coefficient [11]. Every followup scan is registered with the first scan, thereby revealing any possible changes in the microstructure and minimizing errors in the region of interest for the structural parameters.

\section{Mechanical Testing}

After sacrifice, all left tibiae were dissected and used for two different mechanical tests. First, a standard three-point bending test was applied. The tibiae were placed on the lateral surface on two rounded supporting bars with a distance of $2.4 \mathrm{~mm}$. A preload of $1 \mathrm{~N}$ was applied (Z020; Zwick, Ulm, Germany) at the medial surface of the diaphysis by lowering a third rounded bar. A constant displacement rate of $6 \mathrm{~mm} /$ minute was applied until failure. Displacement was measured from the actuator displacement transducer of the testing machine. Stiffness, ultimate force, and ultimate displacement were calculated for each sample.
To assess the mechanical properties of the proximal tibia, a proximal tibia compression test was performed similar to the method of Hogan et al. [12]. A 4-mm slice of the proximal metaphysis including both cortical and trabecular bone was sawed below the growth plate using a diamond saw (Accutom-5; Struers, Ballerup, Denmark). This slice was axially compressed (Z020) during five preconditioning cycles between 1 and $70 \mathrm{~N}$. After the last preconditioning cycle, axial compressive load was applied at $0.5 \mathrm{~mm} / \mathrm{minute}$ until failure, while axial displacement was measured using an extensometer. Stiffness, ultimate force, and ultimate displacement were determined for all samples.

\section{Histomorphometry}

At necropsy, all right tibiae were fixed in $70 \%$ ethanol and embedded in methylmethacrylate (Technovit 9100; Heraeus Kulzer, Wehrheim, Germany). Thin sections (5 $\mu \mathrm{m})$ were cut to determine fluorochrome-based dynamic histomorphometric parameters of bone formation using an Axiophot photomicroscope (Zeiss, Oberkochen, Germany) linked to a camera (CF 15/4 MC; Kappa, Gleichen, Germany), and a QUANTIMET 600 image analysis system was used to calculate the amount of mineralized surface per bone surface (MS/BS, percentage), corrected mineral apposition rate (MAR) $(\mu \mathrm{m} /$ day), and bone formation rate (BFR) per bone surface $(\mu \mathrm{m} /$ day). Calculations of the dynamic parameters were performed as recommended previously [13].

\section{Statistics}

Since this experiment concerned repeated measures in multiple groups, several statistical tests were performed to reveal changes in parameter values over time and between groups. For all structural parameters, the percentage change at different time intervals was calculated for each animal individually. A one-way analyses of variance (ANOVA) with repeated measures was performed on the percentage change for all structural parameters and all groups. Furthermore, a one-way ANOVA with a Bonferroni post-hoc test was used to determine differences between the groups at all time points for each structural parameter. Also, a one-way ANOVA with a Bonferroni post-hoc test was used to determine differences between the groups in cortical thickness, histomorphometric results, and mechanical results. For each group, a paired Student's $t$-test was performed on the absolute values of the structural parameters of each time point compared to baseline values, to determine the earliest point at which a detectable change was found. A paired Student's $t$-test was used to determine 
late ZOL treatment effects within this group. Finally, a paired Student's $t$-test was performed on the absolute values between each measurement for each group and structural parameter. $P<0.05$ was considered significant.

\section{Results}

\section{Ovariectomy}

Based on the uterine weight, we determined seven unsuccessful OVXs. The rats concerned were removed from further analyses, which left nine rats in the control group, five in the OVX group, seven in the OVX and late ZOL group, and eight in the OVX and early ZOL group. For each animal, we determined the percentage change in structural parameters compared to the values at week 0 . Figure 1 shows the average percentage change and upper standard deviation for all groups for
BV/TV, Conn.D, SMI, Tb.N, Tb.Th, and Tb.Sp. The OVX group without treatment showed large changes in structural parameters, indicating the development of OVX-induced bone loss (Figs. 1 and 2). Paired Student's $t$-test indicated significant changes within 2 weeks after OVX for all structural parameters. BV/TV, Conn.D, and Tb.N decreased during the experiment and SMI and Tb.Sp increased. Both Tb.Th and Tb.N decreased within 2 weeks, indicating that both thinning and complete resorption of trabeculae started directly after OVX. The initial rapid loss of bone and connectivity was largely accompanied by trabecular thinning, while secondary, slower loss of bone was concomitant with a decrease in Tb.N. While BV/TV, Conn.D, SMI, Tb.N, and Tb.Sp showed continuous changes in the same direction throughout the experiment, Tb.Th initially decreased significantly until 4 weeks after OVX and then increased again until after 16 weeks $\mathrm{Tb}$.Th was significantly higher than in the control group.
Fig. 1 Average percentage change in structural parameters in the metaphyseal proximal tibia and upper standard deviation for all groups at all time points. Brackets indicate $\mathrm{P}<0.05$ at week 16
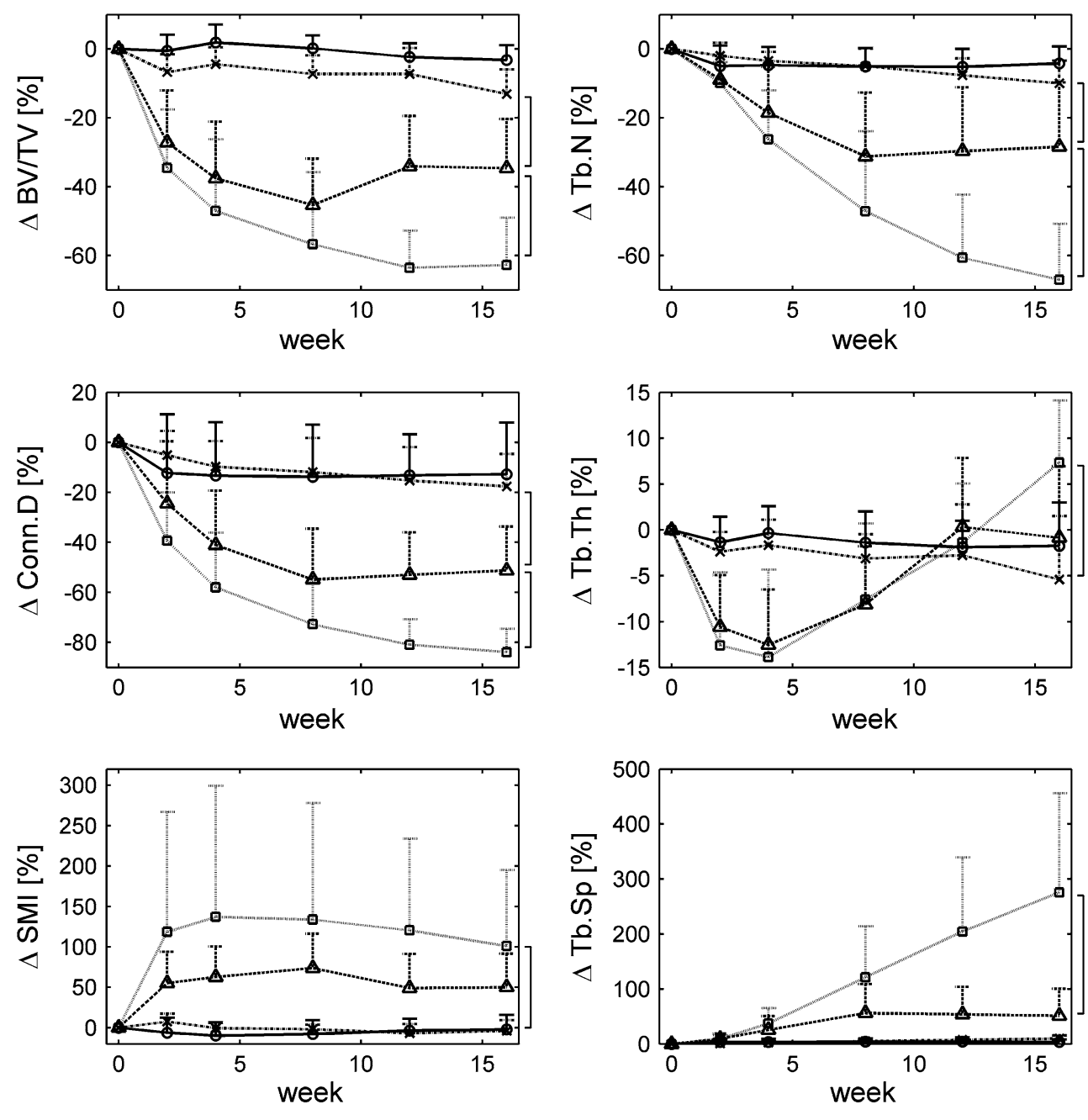

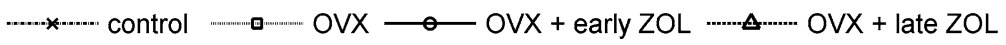



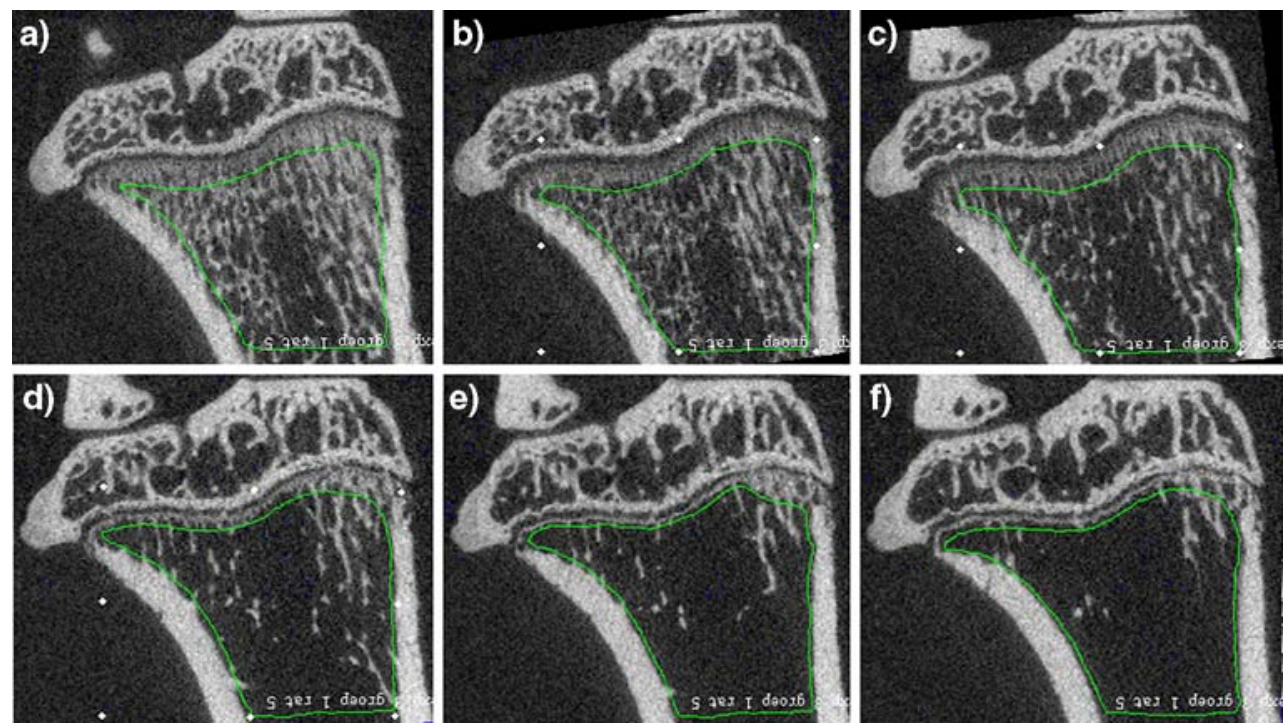

Fig. 2 Same slice of an unprocessed CT scan of the same rat in the OVX group taken at weeks 0 (a), 2 (b), 4 (c), 8 (d), 12 (e), and 16 (f). Images show typical trabecular bone loss due to OVX in the metaphysis. Green line shows the analyzed metaphyseal bone

Table 1 Mean values and standard deviation of all structural parameters of all groups at the start of the experiment

\begin{tabular}{|c|c|c|c|c|c|c|}
\hline Group & $\mathrm{BV} / \mathrm{TV}(1)$ & Conn.D (1/mm) & SMI (1) & Tb.N (1/mm) & Tb.Th $(\mu \mathrm{m})$ & Tb.Sp $(\mu \mathrm{m})$ \\
\hline Control & $0.17(0.056)$ & $41(47)$ & $1.52(0.53)$ & $1.89(1.38)$ & $105(49)$ & $613(368)$ \\
\hline OVX & $0.26(0.055)$ & $141(76)$ & $1.22(0.39)$ & 3.77 (1.66) & $84(10)$ & 335 (174) \\
\hline $\mathrm{OVX}$ + late ZOL & $0.23(0.062)$ & $86(66)$ & $1.23(0.34)$ & $2.77(1.55)$ & $89(13)$ & $482(227)$ \\
\hline OVX + early ZOL & $0.17(0.056)$ & $38(21)$ & $1.70(0.39)$ & $1.86(0.80)$ & $95(7)$ & 647 (207) \\
\hline
\end{tabular}

The absolute average values and standard deviations for all structural parameters of all measurements are shown in Table 1. At the starting point, some differences in the values between the groups were present. This was due to the fact that some animals, which mostly had a relatively low BV/TV, were removed from the study because they did not respond to the OVX. However, since we compared the relative changes in each animal, the results were most likely not affected by the difference in absolute values.

\section{ZOL Treatment}

The early ZOL treatment and control groups were not significantly different at all time points for all structural parameters.

Up until 8 weeks after OVX, when ZOL was administered to rats in the late treatment group, the OVX group and the late treatment group were not significantly different for all structural parameters. BV/TV, SMI, Tb.Th, and Tb.N were significantly improved in the late ZOL group 4 weeks after the single ZOL injection (Table 2). In addition, Tb.Sp significantly improved within 8 weeks after injection,
Table $2 P$ values of paired Student's $t$-test in the late ZOL group after ZOL treatment

\begin{tabular}{lll}
\hline Structural parameter & $P$ for weeks $8-12$ & $P$ for weeks $8-16$ \\
\hline BV/TV & $0.001^{*}$ & $0.004^{*}$ \\
Conn.D & 0.249 & 0.181 \\
SMI & $0.003^{*}$ & $0.004^{*}$ \\
Tb.N & $0.041^{*}$ & $0.005^{*}$ \\
Tb.Th & $0.000^{*}$ & $0.000^{*}$ \\
Tb.Sp & 0.344 & $0.008^{*}$ \\
\hline
\end{tabular}

* Significant $(P<0.05)$

while Conn.D showed no significant response. Between weeks 12 and 16, all structural parameters stayed constant.

At week 16, BV/TV, Conn.D, and Tb.N were significantly different between all groups except for the early treatment and control groups (Table 3). Tb.Th was only significantly different between the control and OVX groups. Although substantial differences in Tb.Sp and SMI were seen between all groups, except for the early treatment and control groups, significant differences were only found between the OVX and control groups and between the OVX and early treatment groups. 
Table $3 P$ values of ANOVA plus Bonferroni post-hoc test comparing all groups at all time points for all structural parameters

\begin{tabular}{|c|c|c|c|c|c|c|c|c|c|c|c|c|c|c|c|}
\hline \multirow[t]{2}{*}{ Group } & \multicolumn{5}{|c|}{$\Delta \mathrm{BV} / \mathrm{TV}$} & \multicolumn{5}{|c|}{$\Delta$ Conn.D } & \multicolumn{5}{|l|}{$\Delta \mathrm{SMI}$} \\
\hline & 2 & 4 & 8 & 12 & 16 & 2 & 4 & 8 & 12 & 16 & 2 & 4 & 8 & 12 & 16 \\
\hline 1 vs. 2 & $0.010^{*}$ & $0.000^{*}$ & $0.000^{*}$ & $0.000^{*}$ & $0.000^{*}$ & $0.025^{*}$ & $0.001^{*}$ & $0.000^{*}$ & $0.000^{*}$ & $0.000^{*}$ & $0.027^{*}$ & $0.008^{*}$ & $0.004^{*}$ & $0.001^{*}$ & $0.003^{*}$ \\
\hline 1 vs. 3 & $0.005^{*}$ & $0.000^{*}$ & $0.000^{*}$ & $0.000^{*}$ & $0.000^{*}$ & 0.341 & $0.021^{*}$ & $0.001^{*}$ & $0.000^{*}$ & $0.005^{*}$ & 0.929 & 0.473 & 0.141 & 0.305 & 0.24 \\
\hline 1 vs. 4 & 0.999 & 0.999 & 0.999 & 0.999 & 0.322 & 0.999 & 0.999 & 0.999 & 0.999 & 0.999 & 0.999 & 0.999 & 0.999 & 0.999 & 0.999 \\
\hline 2 vs. 3 & 0.999 & 0.999 & 0.579 & $0.000^{*}$ & $0.000^{*}$ & 0.999 & 0.906 & 0.811 & $0.042^{*}$ & $0.027^{*}$ & 0.598 & 0.443 & 0.667 & 0.148 & 0.37 \\
\hline 2 vs. 4 & $0.000^{*}$ & $0.000^{*}$ & $0.000^{*}$ & $0.000^{*}$ & $0.000^{*}$ & 0.079 & $0.001^{*}$ & $0.000^{*}$ & $0.000^{*}$ & $0.000^{*}$ & $0.011^{*}$ & $0.005^{*}$ & $0.003^{*}$ & $0.001^{*}$ & $0.003^{*}$ \\
\hline 3 vs. 4 & $0.000^{*}$ & $0.000^{*}$ & $0.000^{*}$ & $0.000^{*}$ & $0.000^{*}$ & 0.9 & $0.023^{*}$ & $0.001^{*}$ & $0.000^{*}$ & $0.000^{*}$ & 0.421 & 0.29 & 0.104 & 0.331 & 0.206 \\
\hline \multirow[t]{2}{*}{ Group } & \multicolumn{5}{|l|}{$\Delta \mathrm{Tb} . \mathrm{N}$} & \multicolumn{5}{|l|}{$\Delta \mathrm{Tb} . \mathrm{Th}$} & \multicolumn{5}{|l|}{$\Delta \mathrm{Tb} . \mathrm{Sp}$} \\
\hline & 2 & 4 & 8 & 12 & 16 & 2 & 4 & 8 & 12 & 16 & 2 & 4 & 8 & 12 & 16 \\
\hline 1 vs. 2 & 0.088 & $0.001^{*}$ & $0.000^{*}$ & $0.000^{*}$ & $0.000^{*}$ & $0.003^{*}$ & $0.002^{*}$ & 0.673 & 0.999 & $0.013^{*}$ & 0.21 & $0.006^{*}$ & $0.001^{*}$ & $0.000^{*}$ & $0.000^{*}$ \\
\hline 1 vs. 3 & 0.108 & $0.019^{*}$ & $0.005^{*}$ & $0.007^{*}$ & $0.018^{*}$ & $0.008^{*}$ & $0.002^{*}$ & 0.325 & 0.999 & 0.999 & 0.099 & 0.072 & 0.2 & 0.802 & 0.999 \\
\hline 1 vs. 4 & 0.999 & 0.999 & 0.999 & 0.999 & 0.999 & 0.999 & 0.999 & 0.999 & 0.999 & 0.999 & 0.999 & 0.999 & 0.999 & 0.999 & 0.999 \\
\hline 2 vs. 3 & 0.999 & 0.999 & 0.353 & $0.001^{*}$ & $0.000^{*}$ & 0.999 & 0.999 & 0.999 & 0.999 & 0.152 & 0.999 & 0.999 & 0.128 & $0.001^{*}$ & $0.000^{*}$ \\
\hline 2 vs. 4 & 0.45 & $0.001^{*}$ & $0.000^{*}$ & $0.000^{*}$ & $0.000^{*}$ & $0.001^{*}$ & $0.001^{*}$ & 0.265 & 0.999 & $0.06^{*}$ & 0.492 & $0.007^{*}$ & $0.001^{*}$ & $0.000^{*}$ & $0.000^{*}$ \\
\hline 3 vs. 4 & 0.623 & $0.027^{*}$ & $0.004^{*}$ & $0.003^{*}$ & $0.002^{*}$ & $0.003^{*}$ & $0.001^{*}$ & 0.109 & 0.999 & 0.999 & 0.279 & 0.084 & 0.187 & 0.667 & 0.999 \\
\hline
\end{tabular}

Groups: 1, control; 2, OVX; 3, late ZOL; 4, early ZOL

* Significant $(P<0.05)$

CT-derived mineralization values in all groups did not change over time and did not differ between groups at all time points.

\section{Aging}

An ANOVA with repeated measures on all structural parameters of the control group was performed to determine if there was a significant effect of aging. BV/TV and Tb.N were found to significantly decrease and Tb.Sp was found to significantly increase due to aging. All other structural parameters did not change significantly in 16 weeks.

An ANOVA with repeated measures was performed on the percentage change in structural parameters comparing the control and early treatment groups to determine any significant differences. BV/TV and Tb.N showed a significant effect of age, treatment, and age * treatment and Tb.Sp showed a significant effect of age and age * treatment. While BV/TV decreased significantly over time in both the early treatment and control groups, it was found to decrease at a higher rate in the latter. Tb.N significantly decreased over time in the control group; however, it did not significantly change in the early treatment group as a net result of OVX, aging, and ZOL treatment. Tb.Sp significantly increased in the control group due to aging, while it did not in the early treatment group.

\section{Cortical Thickness}

OVX initially led to a decrease in metaphyseal cortical thickness, and at 4 weeks both the OVX and the late ZOL groups were significantly lower than the early ZOL group (Fig. 3). However, after 4 weeks it increased again in the OVX group, and at 16 weeks cortical thickness was significantly higher than in the control group. Early ZOL treatment inhibited the changes in cortical thickness. The late ZOL group, just like the OVX group, showed an increase in cortical thickness between 4 and 16 weeks, although this was less pronounced.

\section{Mechanical Testing}

Experimental test results were obtained for seven (control), five (OVX), six (OVX and late ZOL), and seven (OVX and early ZOL) tibiae. No significant differences were found between all parameters determined in the three-point bending test (Fig. 4a).

For the results from the proximal tibia compression test, a significant difference in ultimate displacement was found between the OVX group and the early ZOL group and between the OVX and the control groups (Fig. 4b). Although not significantly determined, a trend was observed in which OVX resulted in a lower stiffness and ultimate force, while late ZOL treatment partially 


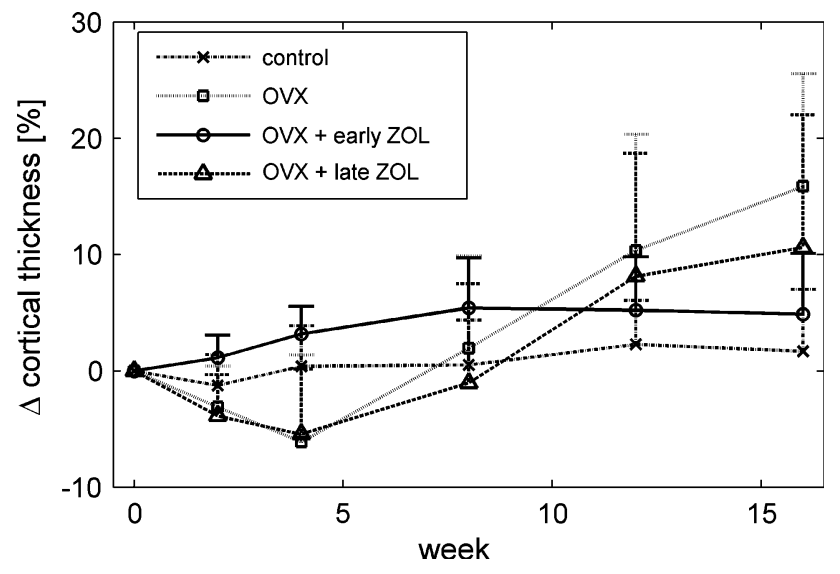

Fig. 3 Average percentage change in cortical thickness in the metaphyseal proximal tibia and upper standard deviation for all groups at all time points. At week 4 the OVX and the late ZOL groups were significantly lower than the early ZOL group, and at week 16 the OVX group was significantly higher than the control group

recovered these values and early ZOL treatment did to a larger extent.

\section{Histomorphometry}

Histomorphometric results were obtained for five (control), five (OVX), six (OVX and late ZOL), and five (OVX and early ZOL) rats. The OVX group showed a significantly higher mineralizing surface (MS/BS) and BFR and a substantially higher mineral apposition rate (MAR) than the control group (Fig. 5). Both ZOL groups showed a significantly lower MS/BS and BFR than the OVX group and control group, while MAR was significantly lower than only the OVX group. Additionally, the late ZOL group had a significantly lower MS/BS and a nonsignificantly lower BFR than the early ZOL group.

\section{Discussion}

In this study we assessed the effects of OVX and of early and late ZOL treatments after OVX on the microstructure of metaphyseal bone in the proximal tibia of aged, female Wistar rats over a 16-week time course, using in vivo highresolution micro-CT.

OVX induced significant changes in all structural parameters within 2 weeks, indicating the rapid development of OVX-induced bone loss. An initial phase of rapid decreases in BV/TV and Conn.D was seen, followed by a second phase in which changes took place at lower rates. The initial rapid loss of bone and connectivity was largely caused by trabecular thinning, while the secondary, slower loss of bone was accompanied by a decrease in Tb.N. OVX induced large decreases in BV/TV, Conn.D, and Tb.N and large increases in Tb.Sp and SMI, indicating the change of more plate- to rod-like bone, which agrees with the literature on the effects of OVX in rat tibiae [2, 14-16]. A different response was, however, seen in Tb.Th, which initially decreased until 4 weeks after OVX and then increased until at the end point of the experiment it was higher than in the control group. A possible explanation for this response could be that, due to increased osteoclast activity, initially most trabeculae will become thinner, while only few are completely resorbed. After a certain period of resorption, the thinner trabeculae will be perforated and completely resorbed. Since the thicker trabeculae remain present, the average $\mathrm{Tb}$.Th will increase. Another possible explanation would be that mechanical adaptation takes place. Since much bone is lost after OVX
Fig. 4 Stiffness, ultimate force, and ultimate displacement determined from three-point bending tests on diaphyseal tibia (a) and axial compression tests (b) on metaphyseal bone. Groups: 1, control; 2, OVX; 3, late ZOL; 4, early ZOL. * Significant difference between groups based on ANOVA and Bonferroni post-hoc test $(P<0.05)$
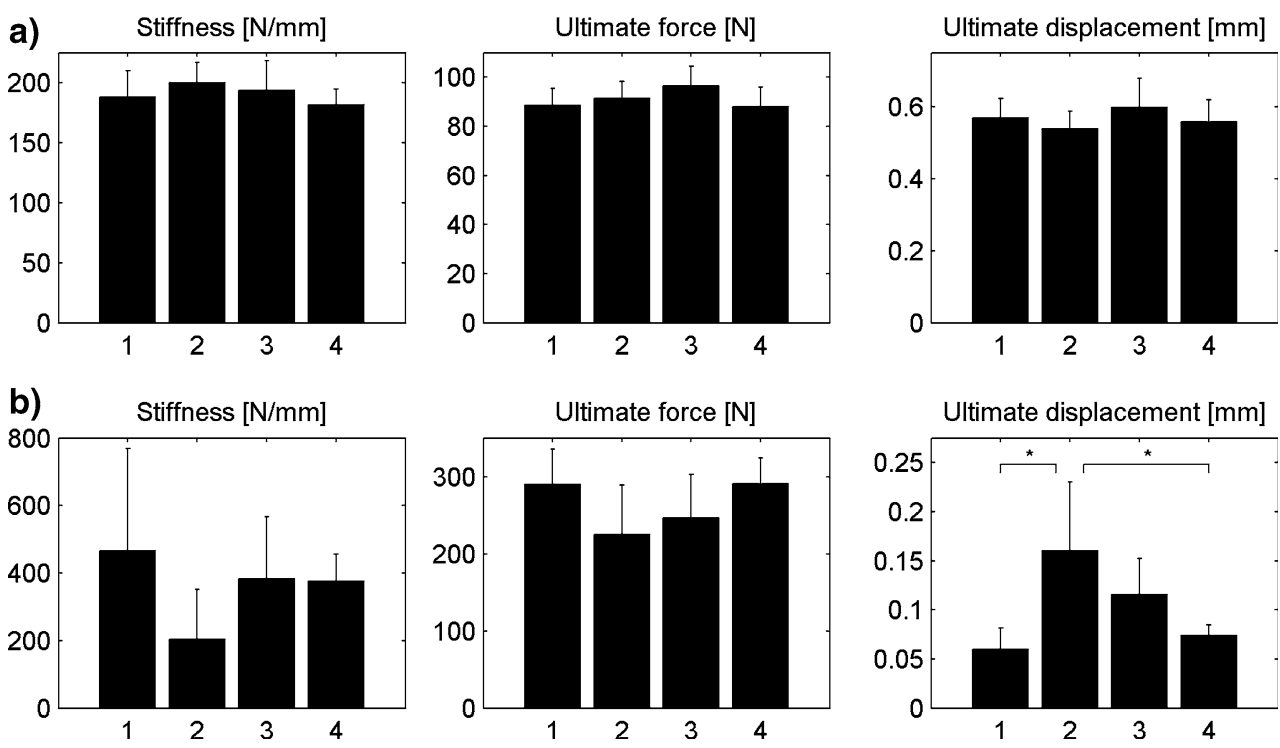

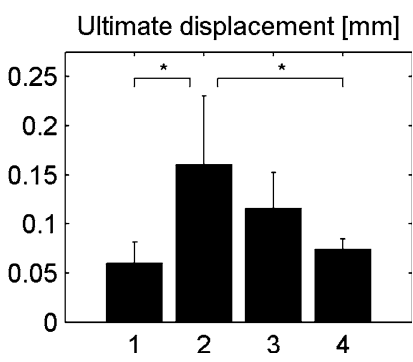



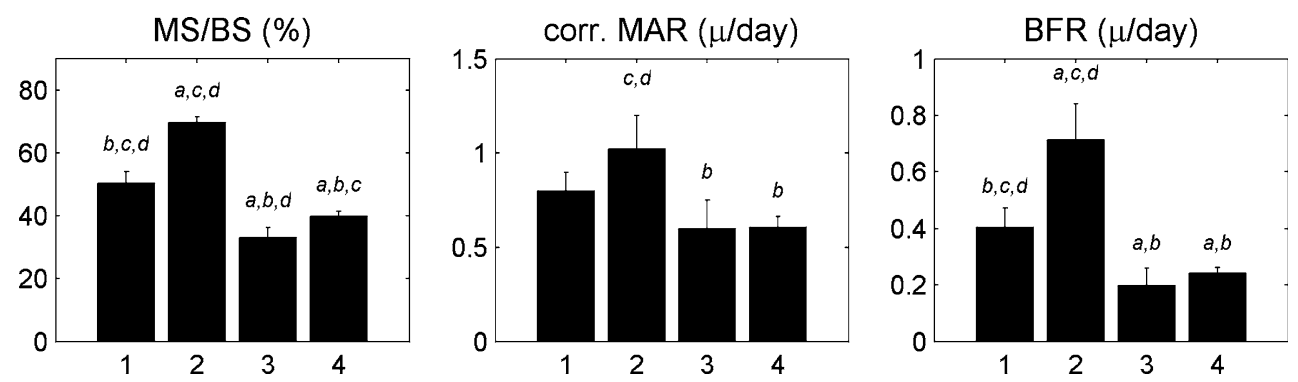

Fig. 5 Dynamic bone histomorphometric parameters for all groups in the metaphyseal proximal tibia. Groups: 1, control; 2, OVX; 3, late ZOL; 4, early ZOL. Based on ANOVA and a Bonferroni test,

and the number and thickness of trabeculae decrease, all load bearing goes through the remaining trabeculae. Therefore, the stress increases in these trabeculae, which stimulates bone formation and would lead to increased $\mathrm{Tb}$.Th. It is also possible that a combination of these mechanisms actually takes place. In the two studies published to date regarding in vivo micro-CT monitoring of the effects of OVX on the metaphyseal proximal tibia in rats, the loss of trabecular bone was similar to what was seen here [5, 7]. However, Tb.Th significantly increased in one study and decreased in the other directly after OVX. Although the same rat strain was used and the same anatomical location was studied, it could be that differences, such as the use of retired breeders vs. virgin rats, age, or type of data analysis, led to variable results.

Early ZOL injection at the time of OVX completely prevented all changes in structural parameters and, thus, the development of OVX-induced bone loss. Aging caused a significant decrease in BV/TV, Conn.D, and Tb.N and an increase in $\mathrm{Tb} . \mathrm{Sp}$ in the control group, which agrees with the literature [7]. However, a significant decrease was merely seen in BV/TV in the early ZOL group and at a significantly lower rate than in the control group. This indicates that a single injection of ZOL was powerful enough to inhibit even natural deterioration of bone volume and microstructure due to aging. One year of weekly ZOL injections of a concentration as low as $0.3 \mu \mathrm{g} / \mathrm{kg}$ completely suppressed the effects of OVX in vertebrae of Sprague-Dawley rats, and $1.5 \mu \mathrm{g} / \mathrm{kg}$ was needed to retain all structural parameters and mechanical properties in the femurs [8, 17]. Similarly, 69 weeks of weekly ZOL injections in OVX rhesus monkeys reduced bone loss in a dose-dependent manner [18]. In a phase II study, both a yearly and a quarterly ZOL dose increased spinal and femoral bone mineral density in postmenopausal women [19]. In the current study, we have shown the longterm, potent inhibiting effects of $\mathrm{ZOL}$ on both BV/TV and the microstructure in rats.

While the preventive effects of ZOL have previously been studied in rats [8, 17], the effect of ZOL treatment after the development of OVX-induced bone loss on the

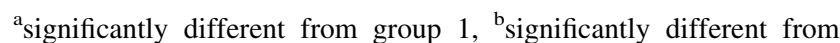
group 2, ${ }^{\mathrm{c}}$ significantly different from group 3 , and $\mathrm{d}_{\text {significantly }}$ different from group $4(P<0.05)$

microstructure, which more closely resembles the clinical situation, is new. Late ZOL injection after 8 weeks of bone loss development inhibited further bone loss. Possibly as a result of the filling of resorption cavities [20], a temporary but significant increase in BV/TV and thickness and a significant decrease in SMI were seen directly after ZOL administration. Four weeks later, however, all structural parameters stayed constant. This is in contrast with the OVX group, which still showed deterioration of bone structure after 8 weeks. Overall, bisphosphonate treatment after the onset of osteoporosis is known to stabilize or increase BV/ TV and Tb.Th in both humans and other animals, which agrees with the data presented here [21-23]. It is generally thought that the underlying mechanism for an increase in $\mathrm{BV} / \mathrm{TV}$ and $\mathrm{Tb}$.Th is the inhibiting effect of bisphosphonates on bone resorption preventing further thinning of trabeculae and the reduction of remodeling space by filling of resorption cavities, leading to increased bone mass [20, 24].

Cortical thickness first decreased as a result of OVX, later increased again, and finally was higher than that of the control group. Both increases and decreases in metaphyseal tibial cortical thickness have been reported in cross-sectional studies, which may be due to different analytic methods or different time points of sacrifice [25, 26]. Early ZOL treatment resulted in a similar cortical thickness as seen in the control groups, which was fairly constant. Late ZOL treatment resulted in a similar pattern as in the OVX group, although less pronounced, which may be explained by the inhibiting effect of ZOL on bone remodeling rate. Cortical thickness profiles in all groups were similar to those for $\mathrm{Tb}$.Th, which may be interpreted as an indication that mechanical adaptation takes place in both cortical and trabecular bone after sustained bone loss.

No significant differences were found in mechanical properties determined in the three-point bending test between the OVX and control groups, which agrees with the literature [27, 28]. Also, no significant influence of ZOL treatment was found on the mechanical properties determined in the three-point bending test, which may be expected as ZOL inhibits bone resorption and does not 
increase formation. In the proximal tibia compression test, ultimate displacement was found to be higher in the OVX group than in the other groups. ZOL treatment partially recovered this increase in ultimate displacement. Although not significantly, ultimate force and stiffness tended to be lower in the OVX group, while ZOL treatment inhibited this. The low number of animals per group and variability in the data unfortunately limit the interpretation of these results. It is known that while endosteal bone resorption takes place during aging and is increased as a result of OVX, periosteal apposition increases as well [29-34], which was also seen in our CT scans (data not shown), thereby compensating for the loss of endocortical and trabecular bone [35]. Therefore, loss of trabecular and endocortical bone may not necessarily mean loss of strength. While first-generation bisphosphonates, such as etidronate, have been shown to directly decrease mineralization of bone $[9,36]$, it is thought that ZOL may increase mineralization by inhibiting resorption and thereby allowing longer mineralization [37, 38]. Furthermore, microdamage could accumulate, and both of these effects could alter the mechanical properties of the bone tissue. Nonetheless, the trends in the mechanical properties resembled the trends in $\mathrm{BV} / \mathrm{TV}$ and showed that $\mathrm{ZOL}$ inhibited the changes resulting from OVX.

Bone-forming activity was slightly higher in the OVX compared to the control group, reflecting an increased turnover rate. Both ZOL groups displayed lower boneforming parameters compared to the control and OVX groups, probably due to suppression of bone formation and resorption. Additionally, the late $\mathrm{ZOL}$ group tended to show slightly less bone-forming activity than the early ZOL group, possibly due to the later time point of treatment. These results agree with previous reports of decreased osteoblast and osteoclast numbers after ZOL injection and decreased number of resorption lacunae [20, 39]. CT-derived estimates of mineralization of metaphyseal trabecular bone were, however, not significantly increased in ZOL-treated rats. This would indicate that ZOL treatment did not influence mineralization in this time frame.

As previously mentioned, at times, substantial differences were found between groups; however, due to a relatively high standard deviation, no significant differences unfortunately were detected. This high standard deviation was mostly seen in the OVX and late treatment group. This was most likely caused by the fact that retired breeders were used, which are known to show a variable response to OVX. Due to an unsuccessful OVX, seven animals were removed from the study. This decreased the number of animals used in the data analysis and, since these were mostly animals with a relatively low BV/TV, the average BV/TV at the start of the study was higher in the OVX and late treatment groups. Therefore, we performed statistical tests on percentage changes in structural parameters rather than on the absolute values. The trend for the percentage and absolute changes looked similar and the $P$ values were similar for most cases. However, when ANOVA was performed on the absolute values comparing all groups at week 16, less significant differences in structural parameters were found, indicating the more sensitive approach of the in vivo experimental design.

Another limitation to this study was the fact that on average, at 8 weeks in the study, the OVX group was in a more deteriorated condition than the late treatment group, while there was no demonstrable explanation for this. Although the ANOVA showed no significant differences between the groups, the treatment group still had a more favorable starting point when ZOL was administered. However, because after ZOL injection significant improvement took place while the OVX group still deteriorated, it is shown that this will not have affected the final conclusions.

The method of in vivo CT scans combined with image registration software presented here has enabled us to study our goals in a more efficient way in terms of number of animals needed in the experiment. Follow-up micro-CT scans were made of the same animals, enabling us to use paired statistics and relative percentage changes in structural parameters. By registering all CT scans, we were able to select the same part of the metaphyseal trabecular bone for every measurement, thereby decreasing the variation in structural parameters due to a varying selected region of interest.

In conclusion, OVX induced rapid changes in trabecular bone mass and structure. A single injection of ZOL at OVX completely inhibited these changes in bone mass and structure seen in osteoporotic rats. A single ZOL injection after OVX-induced bone loss improved bone mass and structure, per group and compared to the control group. $\mathrm{BV} / \mathrm{TV}$ and the associated bone microstructure at the end point of this study were, however, significantly less favorable than in the early treatment group.

Acknowledgement This work was funded by the Netherlands Organization for Scientific Research, Prins Bernard Cultuurfonds, and a VSB-beurs. We thank Jo Habets for performing the OVXs and the animal care.

Open Access This article is distributed under the terms of the Creative Commons Attribution Noncommercial License which permits any noncommercial use, distribution, and reproduction in any medium, provided the original author(s) and source are credited.

\section{References}

1. Dempster DW, Birchman R, Xu R, et al. (1995) Temporal changes in cancellous bone structure of rats immediately after ovariectomy. Bone 16:157-161 
2. Laib A, Kumer JL, Majumdar S, Lane NE (2001) The temporal changes of trabecular architecture in ovariectomized rats assessed by microCT. Osteoporos Int 12:936-941

3. Wronski TJ, Dann LM, Scott KS, Cintron M (1989) Long-term effects of ovariectomy and aging on the rat skeleton. Calcif Tissue Int 45:360-366

4. Lane NE, Thompson JM, Haupt D, et al. (1998) Acute changes in trabecular bone connectivity and osteoclast activity in the ovariectomized rat in vivo. J Bone Miner Res 13:229-236

5. Waarsing JH, Day JS, Verhaar JAN, et al. (2006) Bone loss dynamics result in trabecular alignment in aging and ovariectomized rats. J Orthop Res 24:926-935

6. Yang J, Pham SM, Crabbe DL (2003) Effects of oestrogen deficiency on rat mandibular and tibial microarchitecture. Dentomaxillofac Radiol 32:247-251

7. Boyd SK, Davison P, Muller R, Gasser JA (2006) Monitoring individual morphological changes over time in ovariectomized rats by in vivo micro-computed tomography. Bone 39:854-862

8. Hornby SB, Evans GP, Hornby SL, et al. (2003) Long-term zoledronic acid treatment increases bone structure and mechanical strength of long bones of ovariectomized adult rats. Calcif Tissue Int 72:519-527

9. Theriault RL (2003) Zoledronic acid (Zometa) use in bone disease. Expert Rev Anticancer Ther 3:157-166

10. Brouwers JEM, van Rietbergen B, Huiskes R (2007) No effects of in vivo micro-CT radiation on proximal tibia in Wistar rats detected after eight weekly scans. J Orthop Res 25:1325-1332

11. Verhulp E, Rietbergen Bv, Huiskes R (2004) A three-dimensional digital image correlation technique for strain measurements in microstructures. J Biomech 37:1313-1320

12. Hogan HA, Ruhmann SP, Sampson HW (2000) The mechanical properties of cancellous bone in the proximal tibia of ovariectomized rats. J Bone Miner Res 15:284-292

13. Parfitt AM, Drezner MK, Glorieux FH, et al. (1987) Bone histomorphometry: standardization of nomenclature, symbols, and units. Report of the ASBMR Histomorphometry Nomenclature Committee. J Bone Miner Res 2:595-610

14. Waarsing JH, Day JS, van der Linden JC, et al. (2004) Detecting and tracking local changes in the tibiae of individual rats: a novel method to analyse longitudinal in vivo micro-CT data. Bone 34:163-169

15. Waarsing JH, Day JS, Weinans H (2005) Longitudinal micro-CT scans to evaluate bone architecture. J Musculoskelet Neuronal Interact 5:310-312

16. Wronski TJ, Dann LM, Horner SL (1989) Time course of vertebral osteopenia in ovariectomized rats. Bone 10:295-301

17. Glatt M (2001) The bisphosphonate zoledronate prevents vertebral bone loss in mature estrogen-deficient rats as assessed by micro-computed tomography. Eur Cell Mater 1:18-26

18. Binkley N, Kimmel D, Bruner J, et al. (1998) Zoledronate prevents the development of absolute osteopenia following ovariectomy in adult rhesus monkeys. J Bone Miner Res 13:1775-1782

19. Reid IR, Brown JP, Burckhardt P, et al. (2002) Intravenous zoledronic acid in postmenopausal women with low bone mineral density. N Engl J Med 346:653-661

20. Greiner S, Kadow-Romacker A, Wildemann B, Schwabe P, Schmidmaier G (2007) Bisphosphonates incorporated in a poly(D,L-lactide) implant coating inhibit osteoclast like cells in vitro. J Biomed Mater Res A 83:1184-1191

21. Borah B, Dufresne TE, Chmielewski PA, et al. (2004) Risedronate preserves bone architecture in postmenopausal women with osteoporosis as measured by three-dimensional microcomputed tomography. Bone 34:736-746

22. Ito M (2005) Assessment of bone quality using micro-computed tomography (micro-CT) and synchrotron micro-CT. J Bone Miner Metab 23:115-121

23. Recker R, Masarachia P, Santora A, et al. (2005) Trabecular bone microarchitecture after alendronate treatment of osteoporotic women. Curr Med Res Opin 21:185-194

24. Gasser JA (2006) The relative merits of anabolics versus antiresorptive compounds: where our targets should be, and whether we are addressing them. Curr Opin Pharmacol 6:313-8

25. Bourrin S, Ammann P, Bonjour JP, Rizzoli R (2002) Recovery of proximal tibia bone mineral density and strength, but not cancellous bone architecture, after long-term bisphosphonate or selective estrogen receptor modulator therapy in aged rats. Bone 30:195-200

26. Jiang SD, Shen C, Jiang LS, Dai LY (2007) Differences of bone mass and bone structure in osteopenic rat models caused by spinal cord injury and ovariectomy. Osteoporos Int 18:743-750

27. Kippo K, Hannuniemi R, Lauren L, et al. (1997) Clodronate prevents bone loss in aged ovariectomized rats. Calcif Tissue Int 61:151-157

28. Oxlund H, Andreassen TT (2004) Simvastatin treatment partially prevents ovariectomy-induced bone loss while increasing cortical bone formation. Bone 34:609-618

29. Aerssens J, van Audekercke R, Talalaj M, et al. (1996) Effect of 1alpha-vitamin $\mathrm{D}_{3}$ and estrogen therapy on cortical bone mechanical properties in the ovariectomized rat model. Endocrinology 137:1358-1364

30. Danielsen CC, Mosekilde L, Svenstrup B (1993) Cortical bone mass, composition, and mechanical properties in female rats in relation to age, long-term ovariectomy, and estrogen substitution. Calcif Tissue Int 52:26-33

31. Day CJ, Kim MS, Stephens SRJ, et al. (2004) Gene array identification of osteoclast genes: differential inhibition of osteoclastogenesis by cyclosporin A and granulocyte macrophage colony stimulating factor. J Cell Biochem 91:303-315

32. Ke HZ, Jee WS, Zeng QQ, et al. (1993) Prostaglandin $E_{2}$ increased rat cortical bone mass when administered immediately following ovariectomy. Bone Miner 21:189-201

33. Pan Z, Jee WSS, Ma YF, et al. (1995) Intermittent treatments of prostaglandin $E_{2}$ plus risedronate and prostaglandin $E_{2}$ alone are equally anabolic on tibial shaft of ovariectomized rats. Bone 17:S291-S296

34. Turner RT, Vandersteenhoven JJ, Bell NH (1987) The effects of ovariectomy and 17 beta-estradiol on cortical bone histomorphometry in growing rats. J Bone Miner Res 2:115-122

35. Riggs BL, Melton Iii LJ, Robb RA, et al. (2004) Population-based study of age and sex differences in bone volumetric density, size, geometry, and structure at different skeletal sites. J Bone Miner Res 19:1945-1954

36. Kitauchi T, Yoshida K, Yoneda T, et al. Association between pentosidine and arteriosclerosis in patients receiving hemodialysis. Clin Exp Nephrol 8:48-53

37. Little DG, McDonald M, Sharpe IT, et al. (2005) Zoledronic acid improves femoral head sphericity in a rat model of perthes disease. J Orthop Res 23:862-868

38. Pan B, To LB, Farrugia AN, et al. (2004) The nitrogen-containing bisphosphonate, zoledronic acid, increases mineralisation of human bone-derived cells in vitro. Bone 34:112-123

39. Herrak P, Gortz B, Hayer S, et al. (2004) Zoledronic acid protects against local and systemic bone loss in tumor necrosis factormediated arthritis. Arthritis Rheum 50:2327-2337 